\title{
Achieving High Efficiency and Pure Blue Color in Hyperfluorescence Organic Light Emitting Diodes using Organo-Boron Based Emitters
}

Kenkera Naveen

Kyung Hee University https://orcid.org/0000-0002-1067-0740

Hyuna Lee

Kyung Hee University

Ramanaskanda Braveenth

Kyung Hee University

Durai Karthik

Kyung Hee University https://orcid.org/0000-0003-1243-7464

Ki Yang

Kyung Hee University

\section{Soon Hwang}

Kyung Hee University

Jang Hyuk Kwon ( $\nabla$ jhkwon@khu.ac.kr)

Kyung Hee University https://orcid.org/0000-0002-1743-1486

\section{Article}

Keywords: DABNA, boron blue TADF, FRET, Hyperfluorescence, OLED

Posted Date: May 28th, 2021

DOl: https://doi.org/10.21203/rs.3.rs-537019/v1

License: (c) (1) This work is licensed under a Creative Commons Attribution 4.0 International License. Read Full License

Version of Record: A version of this preprint was published at Advanced Functional Materials on December 16th, 2021. See the published version at https://doi.org/10.1002/adfm.202110356. 


\title{
Achieving High Efficiency and Pure Blue Color in Hyperfluorescence Organic Light Emitting Diodes using Organo-Boron Based Emitters
}

Kenkera Rayappa Naveen ${ }^{1}$, Hyuna Lee ${ }^{1}$, Ramanaskanda Braveenth, Durai Karthik, Ki Joon Yang, Soon Jae Hwang and Jang hyuk kwon*

${ }^{1}$ Theses authors contributed equally.

Organic Optoelectronic Device Lab (OODL), Department of Information Display, Kyung Hee University, 26, Kyungheedae-ro, Dongdaemun-gu, Seoul, 02447, Republic of Korea

\section{E-mail: jhkwon@khu.ac.kr}

\begin{abstract}
In the field of organic light emitting diodes (OLEDs), organo-boron based thermally activated delayed fluorescence (TADF) emitters have reached great achievement. However, it is still challenging to achieve pure blue color (CIE y <0.20) along with high efficiencies. To overcome these hurdles, hyperfluorescence (HF) suggest a key strategy in future OLED applications. Here, we report two TADF materials, $p$ MDBA-DI and $m$ MDBA-DI. Further, a pure blue multi resonance type tert-butyl substituted fluorescence emitter, $t$-Bu- $v$-DABNA was also synthesized. Among our synthesized TADF materials based pure blue HF devices, $m$ MDBA-DI as TADF sensitized host with $t$-Bu- $v$-DABNA fluorescence emitter exhibited a high external quantum efficiency of $40.7 \%$ (Lambertian assumption) along with narrow emission with full width at half maximum of $19 \mathrm{~nm}$ $(\mathrm{CIE} \mathrm{y}=0.15)$. Moreover, we analyzed that such high device efficiency is mainly attributed to the high orientation factor, enhanced photoluminescence quantum yield, and a good TADF characteristic of $t$-Bu- $v$-DABNA with high Förster resonance energy transfer.
\end{abstract}

Keyword: DABNA, boron blue TADF, FRET, Hyperfluorescence, OLED 


\section{Introduction}

In recent years, thermally activated delayed fluorescence (TADF) emitters have drawn great interest in the field of organic light emitting diodes (OLEDs). TADF emitters can achieve 100\% internal quantum efficiency (IQE) by utilizing the dark triplet excitons through reverse intersystem crossing pathway. Which is a key advantage over low efficiency conventional fluorescence emitters, where it was able to achieve $25 \%$ IQE using only singlet excitons. Moreover, TADF molecules are using inexpensive organic moieties compared to noble metal complex-based

phosphorescence emitters. ${ }^{1-4}$ Generally, TADF emitters exhibit well separated frontier molecular orbitals due to their donor (D) and acceptor (A) molecular configuration. ${ }^{5-7}$ The well separated highest occupied molecular orbital (HOMO) and lowest unoccupied molecular orbital (LUMO) tend to minimizes electron exchange energy $(J)$, resulting in a small singlet $\left(\mathrm{S}_{1}\right)$ - triplet $\left(\mathrm{T}_{1}\right)$ energy gap $\left(\Delta \mathrm{E}_{\mathrm{ST}}\right)$ with good charge transfer $(\mathrm{CT})$ characteristics accompanied by delayed emission. ${ }^{8}$ However, such CT characteristics of TADF emitters were reported to have wide full width halfmaximum (FWHM) of $\geq 50 \mathrm{~nm}$, which makes limitation on high color purity applications. Moreover, the excitons on TADF emitters are long-lived and may easily lead to material degradation followed by efficiency drop at high luminescence region. ${ }^{9}$ Even though, there have been many reports showing high electroluminescence efficiencies, still challenging to produce good color purity satisfying required Commission Internationale de l'Éclairage (CIE) coordinates. In order to overcome above issues, Adachi et al. proposed a future generation technology called hyperfluorescence (HF) OLEDs, and it revealed high efficiency along with high color purity. ${ }^{9}$ In this approach we should take three elements into account, which are TADF emitters, fluorescent emitters with narrow FWHM and high triplet energy host material. To activate the HF system, a fast Förster resonance energy transfer (FRET) from the singlet excited $\left(\mathrm{S}_{1}\right)$ state of TADF molecule 
to the $S_{1}$ of fluorescent emitter is inevitable. Through fast FRET, fluorescent emitter can harvest large amount of singlet excitons coming from the TADF molecule, and emits pure fluorescence emission. Previous studies indicate that such fast FRET leads to reduce the exciton lifetime of TADF molecule while enhancing the device stability. ${ }^{10-15}$ From this HF technology, we can increase the device efficiencies, operational lifetime and good CIE coordinates with narrow emission spectrum. Recently, Hatakeyama et al. reported multi-resonance (MR) type of TADF emitter named $v$-DABNA, which showed high maximum EQE of $34.4 \%$ in blue region with extremely narrow FWHM of $18 \mathrm{~nm} .{ }^{16}$ Thus, many researchers utilized $v$-DABNA as a blue fluorescent emitter in HF-OLEDs to obtain good efficiencies along with pure color and narrow FWHM. Out of many studies related to HF, Adachi et al. reported pure-blue HF device exhibiting maximum EQE of $27 \%$ with CIE y of $0.20 .{ }^{17}$ Consequently, Lee et al. reported triplet-excitondistributed TADF devices with high maximum EQE of 33.5\%.$^{18}$ However, both HF devices limited the device efficiency and the CIE y coordinate due to insufficient FRET energy transfer resulted from less spectral overlap between the TADF emission and the absorption of fluorescent emitter. So, there is a highly requirement in developing efficient deep blue TADF emitters to enhance the FRET energy transfer. In order to achieve deep blue color around 450 $460 \mathrm{~nm}$ and high efficiency simultaneously, boron acceptor based TADF emitters could be promising candidates..$^{7-8,} 19$ Previously reported our emitter, namely 5-(5,9-dioxa-13b boranaphtho[3,2,1de]anthracen-7-yl)-10,15-diphenyl-10,15-dihydro-5H-diindolo[3,2-a:3',2'-c] carbazole (DBA-DI) manifested long device lifetime and high efficiency of $28.1 \%$. However, it showed emission in the sky-blue region (CIE $\mathrm{y}=0.38) .{ }^{20}$ To get emission in the region of $450 \sim 460 \mathrm{~nm}$, certain modification on DBA-DI moiety are required for high efficiency and pure blue HF-OLEDs. Then, 
it is expected that modified TADF host with $v$-DABNA type final emitter can surpass the EQE over $35 \%$ with pure blue color emission.

By considering all discussed parameters, herein we report two new blue TADF emitters namely, 5-(2,12-dimethyl-5,9-dioxa-13b-boranaphtho[3,2,1-de]anthracen-7-yl)-10,15-diphenyl-10,15dihydro-5H-diindolo[3,2-a:3',2'-c]carbazole (pMDBA-DI) and 5-(3,11-dimethyl-5,9-dioxa-13bboranaphtho[3,2,1-de]anthracen-7-yl)-10,15-diphenyl-10,15-dihydro-5H-diindolo[3,2-a:3',2'c]carbazole (mMDBA-DI). Additionally, we have designed and synthesized narrow MR type fluorescence emitter N7,N7,N13,N13,5,15-hexaphenyl-9,11-bis(4-(tert-butyl)phenyl)-5,9,11,15tetrahydro-5,9,11,15-tetraaza-19b,20b-diboradinaphtho [3,2,1-de:1',2',3'-jk]pentacene-7,13diamine ( $t$-Bu- $v$-DABNA). Both $p$ MDBA-DI and $m$ MDBA-DI were incorporated using oxygen bridged triaryl-boron based acceptors, $p$ MDBA (methyl substitution on oxygen para) and $m$ MDBA (methyl substitution on oxygen meta position) with DI donor moiety. The new narrow emitter $t$ $\mathrm{Bu}-v$-DABNA was prepared by attaching tert-butyl group to the reported $v$-DABNA core. The photophysical, electrochemical and device properties of all these TADF and fluorescent emitter were investigated for the HF-OLEDs. We achieved maximum EQE of $32.0 \%$ for $p$ MDBA-DI based TADF device, and HF device using $m \mathrm{MDBA}-\mathrm{DI}$ as TADF assistant host brought excellent EQE over 39.1\%. According to our study, we confirm that such high efficiencies are originated by a high orientation factor, enhanced photoluminescence quantum yield (PLQY), and a good TADF characteristic of final fluorescent emitter and effective FRET energy transfer from TADF assistant host to the emitter.

\section{Results}

\section{Design strategy and theoretical calculations}


Considering the previous reports based on boron acceptors, the symmetrical oxygen bridged boron acceptor was one of the promising acceptor candidates for deep blue TADF materials due to its weak acceptor strength, high PLQY and high RISC rate. ${ }^{79}$ 19-22 Even though previously reported emitter DBA-DI exhibits high efficiency and long device lifetime, the emission color is too much red shifted, $\left(\lambda_{\mathrm{em}}=467 \mathrm{~nm}\right),{ }^{20}$ which makes insufficient energy transfer to pure-blue HF system. Thus, we modified only the acceptor unit by introducing weak electron donating groups on DBA moiety, and we can anticipate that acceptor strength can be reduced while broadening the optical band gap. The newly designed boron acceptors, $p$ MDBA (methyl groups on the para-) and $m$ MDBA (meta- position of oxygen) were connected with DI donor to develop our new TADF emitters, namely $p$ MDBA-DI and $m$ MDBA-DI, respectively. In HF system, attaching bulky or inert group on fluorescent emitters can reduce the unwanted Dexter energy transfer (DET). Usually, incorporation of tert-butyl groups on the terminal sites of the molecules can effectively prohibit the DET and increase the PLQY by reducing the aggregation-caused self-quenching. Thus, we attached tert-butyl groups on $v$-DABNA core to increase the intermolecular distance with nearby molecules and enhance the PLQY. Eventually, we designed $t$-Bu-v-DABNA as novel blue MR type emitter for high color purity in HF system.

To support our design strategy and investigate the photophysical, electronic properties of designed molecules, the density functional theory (DFT) calculation for the optimization of the ground state, and time-dependent DFT (TD-DFT) calculation for the excited state were performed. The calculations were obtained by utilizing the Lee-Yang-Parr correlation function (B3LYP) with 631 (G) basis set using Schrödinger 2020-4 program. ${ }^{23}$ As illustrated on Figure S1, it is clear that the HOMO and LUMO are well-separated, in which HOMO was mainly distributed over the DI donor and LUMO was located on MDBA acceptor, leading to good charge transfer characteristics 
and small $\Delta \mathrm{E}_{\mathrm{ST}}$. The calculated HOMO and LUMO energy values were 5.50 and $2.69 \mathrm{eV}$ for pMDBA-DI, 5.49 and $2.65 \mathrm{eV}$ for $m$ MDBA-DI, respectively. As expected, the HOMO energy level was similar due to the presence of same donor moiety (DI), while LUMO energy level of $p$ MDBA-DI and $m$ MDBA-DI became shallower compared to DBA-DI due to methyl substitution on the boron acceptors. The optical band gap was calculated as 2.81 and $2.84 \mathrm{eV}$ for $p$ MDBA-DI and $m$ MDBA-DI, respectively. Thus, we can expect that both $p$ MDBA-DI and $m$ MDBA-DI will show blue shifted emission compared to DBA-DI. The calculated singlet and triplet energies are 3.03/2.94 eV for $p$ MDBA-DI, and 2.95/3.09 eV for $m$ MDBA-DI, respectively. The calculated $\Delta \mathrm{E}_{\text {ST }}$ values are $0.09 \mathrm{eV}$ and $0.14 \mathrm{eV}$ for $p$ MDBA-DI and $m$ MDBA-DI, respectively. These small $\Delta \mathrm{E}_{\mathrm{ST}}$ energy values indicate that $p \mathrm{MDBA}-\mathrm{DI}$ and $m \mathrm{MDBA}-\mathrm{DI}$ could reveal good TADF performances. Additionally, the theoretical calculations were performed for $t$-Bu- $v$-DABNA and $v$-DABNA and compared each. $t$-Bu- $v$-DABNA exhibits atomically separated frontier molecular orbital (FMO) distributions similar to $v$-DABNA, where HOMO distributes mainly on nitrogen atoms, and LUMO locates primarily on boron atoms as shown in Figure S2. These alternative distributions clearly indicate that it is an effective MR molecular structure. The calculated energy levels of $t$-Bu- $v$-DABNA and $v$-DABNA were almost similar. Thus, we can expect that $t$-Bu- $v$ DABNA also will show similar properties.

\section{Material Synthesis}

The synthesis of $p$ MDBA-DI, $m$ MDBA-DI, and MR type $t$-Bu-v-DABNA is shown in Scheme 1. The new acceptors ( $p$ MDBA-Br and $m \mathrm{MDBA}-\mathrm{Br}$ ) were synthesized by using two-step process which includes cyclization in the presence of $n$-butyllithium $(n-\mathrm{BuLi})$ and boron tribromide $\left(\mathrm{BBr}_{3}\right)$. The final compounds ( $p$ MDBA-DI and $m$ MDBA-DI) were synthesized by using palladium catalyzed Buchwald-Hartwig amination reaction between diindolocarbazole donor (DI) and boron 
acceptors ( $p$ MDBA-Br and $m$ MDBA-Br). And $t$-Bu- $v$-DABNA MR emitter was synthesized by using commercially available compounds in two steps process, which include palladium catalyzed C-N coupling and one-pot double borylation. The detailed synthetic procedures are given in the supplementary information. The structures of all the synthesized materials were clearly characterized by ${ }^{1} \mathrm{H}$ and ${ }^{13} \mathrm{C}$ NMR and high-resolution mass spectrometry (HRMS) (Figure S13S28).
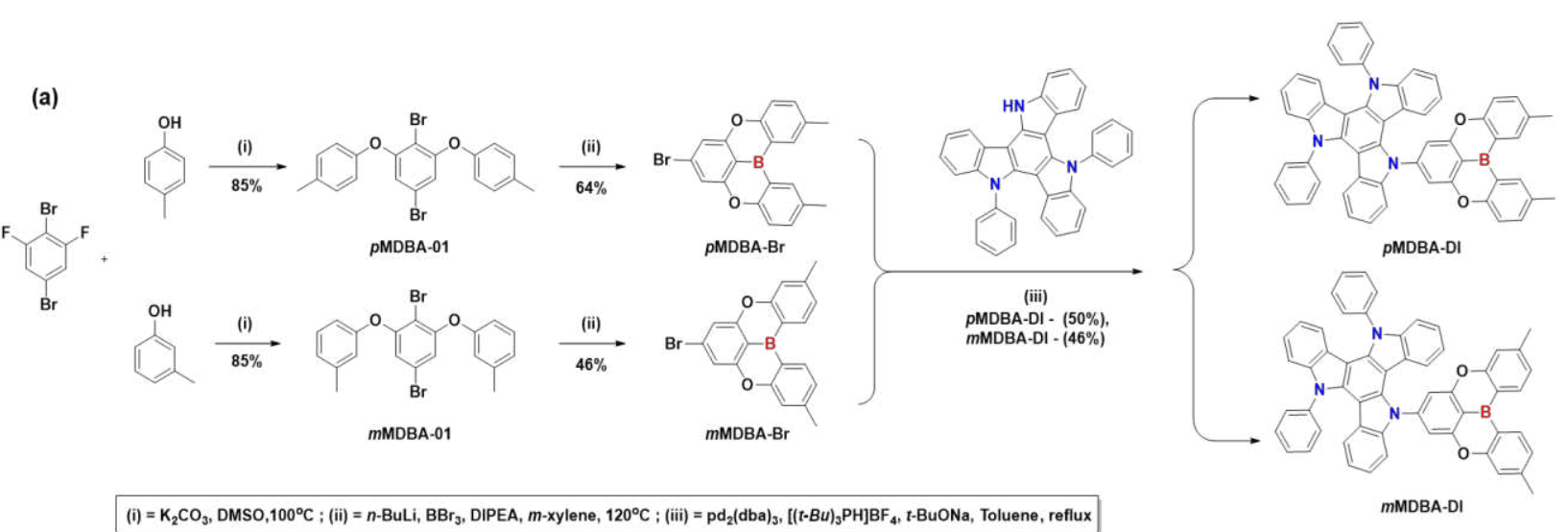

(b)
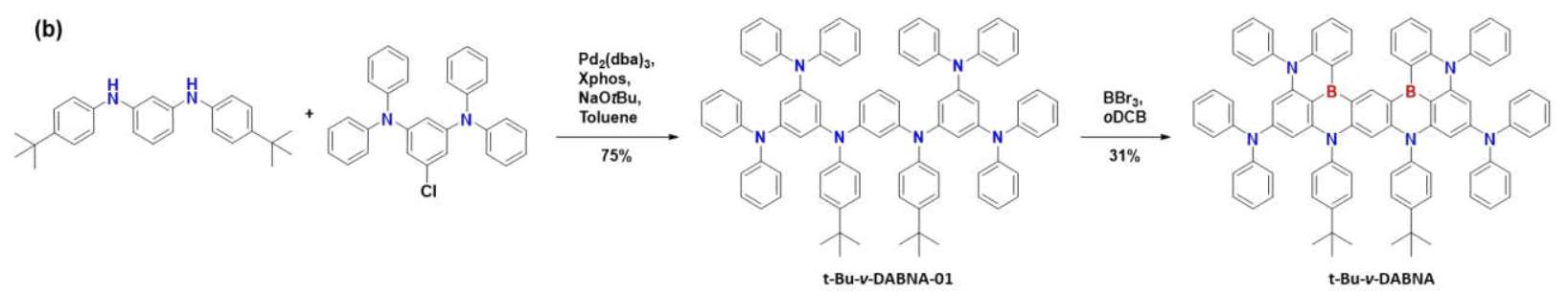

Scheme 1. Synthetic routes and structures of (a) $p$ MDBA-DI, $m$ MDBA-DI and (b) $t-\mathrm{Bu}-v-$ DABNA.

\section{Electrochemical properties}

To understand the electrochemical properties of the synthesized materials, cyclic voltammetry (CV) measurement was performed, and the HOMO energy level was obtained from the oxidation potential. The LUMO energy level was determined by subtracting the energy level of HOMO and optical band gap. The calculated HOMO values from the CV were $5.50 \mathrm{eV}$ for $p$ MDBA-DI and 
$5.48 \mathrm{eV}$ for $m$ MDBA-DI. The optical band gap $\left(\mathrm{E}_{\mathrm{g}}\right)$ calculated from the absorption onset was 2.89 and $2.91 \mathrm{eV}$ for $p$ MDBA-DI and $m$ MDBA-DI, respectively. The calculated LUMO values were 2.61 and $2.57 \mathrm{eV}$ for $p$ MDBA-DI, $m$ MDBA-DI, respectively, which exhibited shallower LUMO energy level than DBA-DI $(2.71 \mathrm{eV})^{20}$. Particularly, mMDBA-DI showed $0.04 \mathrm{eV}$ shallower LUMO energy level than $p$ MDBA-DI as expected in DFT calculation. Since HOMO energy levels were similar among three materials, optical band gaps were broadened compared to DBA-DI. All electrochemical measurement data of TADF materials was well-corresponded with the DFT calculation. In case of $t$-Bu-v-DABNA, the calculated HOMO and LUMO energy levels were 5.54 and $2.94 \mathrm{eV}$, and optical band gap was $2.60 \mathrm{eV}$. According to our electrochemical measurement of $v$-DABNA, the band gap energy level was the same but, HOMO and LUMO energy level became deeper by $0.03 \mathrm{eV}$. The corresponding graphs of all cyclic voltammetry (CV) for all materials are depicted in Figure S3.

\section{Photophysical properties}

To investigate the photophysical properties of synthesized materials, ultraviolet-visible (UV-vis) absorption and photoluminescence (PL) spectra were recorded in toluene solution. Roomtemperature photoluminescence (RTPL) spectra was measured at $300 \mathrm{~K}$ and low-temperature photoluminescence (LTPL) spectra was measured at $77 \mathrm{~K}$ after $30 \mathrm{~ms}$ of delay from the excitation. In UV-Vis absorption, $\pi-\pi^{*}$ transitions were occurred from diindolocarbazole (DI) donor moiety around $320 \mathrm{~nm}$ in both $p$ MDBA-DI and $m$ MDBA-DI. In addition, intramolecular charge transfer (ICT) induced $\mathrm{n}-\pi^{*}$ absorption was appeared at $384 \mathrm{~nm}$ for $p$ MDBA-DI and $371 \mathrm{~nm}$ for $m$ MDBADI, respectively. The PL emission spectra exhibited at 460 and $451 \mathrm{~nm}$ for $p$ MDBA-DI and $m$ MDBA-DI, respectively. As expected, both new TADF materials obtained hypsochromic-shifted emission compared to $467 \mathrm{~nm}$ of DBA-DI. ${ }^{20}$ It is attributed that incorporation of methyl groups in 
boron acceptor could weaken the acceptor strength as we mentioned above, and increase the band gap effectively. Especially, $m$ MDBA-DI emitter showed $16 \mathrm{~nm}$ blue shifted emission from DBADI. Both materials revealed positive solvatochromism effect from non-polar $n$-hexane to polar methylene chloride solvent, and the emission peaks were shifted by $111 \mathrm{~nm}$ (pMDBA-DI) and 107 nm ( $m$ MDBA-DI), respectively. (Figure S4) This solvatochromism results indicate that both materials possess strong ICT of donor-acceptor interaction. Consequently, we can observe that weaker acceptor of $m \mathrm{MDBA}-\mathrm{DI}$ exhibited less solvatochromism compared to $p \mathrm{MDBA}-\mathrm{DI}$. The $\mathrm{S}_{1}$ and $\mathrm{T}_{1}$ energy values were determined by calculating the onset point of the RTPL and LTPL spectra, respectively. The $\mathrm{S}_{1}$ and $\mathrm{T}_{1}$ values were $2.99 / 2.92 \mathrm{eV}$ for $p$ MDBA-DI and 3.04/2.92 eV for $m$ MDBA-DI, respectively. The calculated $\Delta \mathrm{E}_{S T}$ values were 0.07 and $0.12 \mathrm{eV}$ for $p$ MDBA-DI and $m$ MDBA-DI. Further, photophysical properties of $t$-Bu- $v$-DABNA were measured, and the maximum absorption peak was at $457 \mathrm{~nm}$ and PL emission peak was notice at $467 \mathrm{~nm}$ with narrow FWHM of $14 \mathrm{~nm}$ in toluene solution. $t$-Bu-v-DABNA exhibited small solvatochromisim effect, shifting only $14 \mathrm{~nm}$ from hexane to methylene chloride. These narrow FWHM and small Stoke's shift are attributed to the alternative FMO distribution on $t$-Bu- $v$-DABNA core due to non-bonding characters, and which minimizes the vibronic coupling between the $S_{0}$ and $S_{1}$ states, and the vibrational relaxation at the $S_{1}$ state. ${ }^{16}$ The $S_{1}$ and $T_{1}$ energy values were calculated to be 2.78 and $2.74 \mathrm{eV}$, respectively, and $\Delta \mathrm{EST}_{S \mathrm{~T}}$ value is $0.04 \mathrm{eV}$. The corresponding spectra are shown in Figure 1, and the details of the photophysical and electrochemical data of all materials are summarized in

Table 1.

In order to confirm the TADF behavior of $p$ MDBA-DI and $m$ MDBA-DI, transient-PL (TRPL) measurements were performed by using two different solvents, toluene and methylene chloride as shown in Figure 1. Both materials exhibited clear prompt and delayed decay curves, which 
confirms the TADF characteristics. In toluene, the measured prompt $\left(\tau_{\mathrm{p}}\right)$ exciton lifetimes were 24.6 and $28.0 \mathrm{~ns}$ for $p$ MDBA-DI and $m \mathrm{MDBA}-\mathrm{DI}$, respectively. Additionally, calculated delayed $\left(\tau_{\mathrm{d}}\right)$ exciton lifetimes were 1.60 and $1.90 \mu$ s for $p$ MDBA-DI and $m$ MDBA-DI, respectively. Especially, both exhibited short delayed $\left(\tau_{\mathrm{d}}\right)$ exciton lifetimes of 0.75 and $0.80 \mu$ s in methylene chloride. This phenomenon is due to the stabilization of $S_{1}$ state in polar medium. In order to verify the TADF performance in the film state, PLQY and TRPL measurements were recorded in $30 \%$ doped $p$ MDBA-DI and $m$ MDBA-DI with dibenzo[ $[b, d]$ furan-2,8-diylbis(diphenylphosphine oxide) (DBFPO) host. The PLQY values in DBFPO film were 97.8 and $97.3 \%$ for $p$ MDBA-DI and $m$ MDBA-DI emitters, respectively. In addition, the delayed lifetime in DBFPO film still exhibited the same tendency with solution results, which were 1.80 and $2.90 \mu$ s for $p$ MDBA-DI and $m$ MDBA-DI, respectively. Furthermore, to analyze the TADF characteristics of both materials quantitatively, we calculated the various rate constants of excited state processes by using reported method. ${ }^{24}$ Calculated rate constants are listed in Table S1. Among them, the $p$ MDBA-DI exhibited high $k_{\text {RISC }}$ of $1.14 \times 10^{6} \mathrm{~s}^{-1}$ and almost 2 times higher than $6.11 \times 10^{5} \mathrm{~s}^{-1}(\mathrm{mMDBA}-\mathrm{DI})$ and which is due to small $\Delta \mathrm{E}_{\mathrm{ST}}$ values and short delayed lifetime of $p$ MDBA-DI. Although their $k_{\mathrm{RISC}}$ values were lower than DBA-DI ${ }^{20}$, these are still high enough to have similar TADF mechanism. Moreover, we investigated the TRPL and PLQY of $t$-Bu- $v$-DABNA, and measured prompt exciton lifetime $\left(\tau_{\mathrm{p}}\right)$ and delayed exciton lifetime $\left(\tau_{\mathrm{d}}\right)$ in toluene were $5.79 \mathrm{~ns}$ and $1.55 \mu \mathrm{s}$, respectively while delayed exciton lifetime $\left(\tau_{\mathrm{d}}\right)$ in methylene chloride became longer as $1.88 \mu \mathrm{s}$. In film state, $5 \%$ doping of $t$-Bu- $v$-DABNA in DBFPO host exhibited enhanced PLQY value of 91.9\% compared to $v$-DABNA, such enhancement is due to the incorporation of tert-butyl group. In TRPL measurement, $t$-Bu- $v$-DABNA showed short delayed exciton lifetime of $2.93 \mu \mathrm{s}$, and $k_{\mathrm{RISC}}$ was $2.54 \times 10^{5} \mathrm{~s}^{-1}$, which is lower than other TADF materials. Thus, TADF performance expected 
to be relatively poor. On the other hand, extremely narrow FWHM and high PLQY of $t$-Bu- $v$ DABNA would be good characteristics as fluorescence emitter in HF system. All measured film TRPL decay lifetime and calculated rate constants are displayed in Figure S5 and summarized

\section{Table S1.}

\section{Thermal properties}

The thermal properties of our synthesized materials were investigated by using the thermogravimetric analysis (TGA) and differential scanning calorimetry (DSC). The decomposition temperatures $\left(\mathrm{T}_{\mathrm{d}}\right)$ of the materials were measured at $5 \%$ weight reduction, and the observed values were $447{ }^{\circ} \mathrm{C}(p \mathrm{MDBA}-\mathrm{DI}), 470^{\circ} \mathrm{C}(\mathrm{mMDBA}-\mathrm{DI})$ and $520^{\circ} \mathrm{C}(t$-Bu- $v$-DABNA $)$, respectively. The glass transition temperature $\left(\mathrm{T}_{\mathrm{g}}\right)$ was obtained by using DSC measurement. The $\mathrm{T}_{\mathrm{g}}$ values are 226 ${ }^{\circ} \mathrm{C}(p \mathrm{MDBA}-\mathrm{DI}), 232^{\circ} \mathrm{C}(m \mathrm{MDBA}-\mathrm{DI})$ and $273{ }^{\circ} \mathrm{C}(t-\mathrm{Bu}-v$-DABNA $)$, respectively. These results suggest that all these materials revealed high thermal stabilities. All corresponding TGA and DSC figures were shown in Figure S6-S7.

Table 1. The photophysical, electrochemical properties of $p$ MDBA-DI, $m$ MDBA-DI and $t$-Bu- $v$ DABNA.

\begin{tabular}{|c|c|c|c|c|c|c|c|c|c|c|c|c|}
\hline & $\begin{array}{c}\lambda_{\mathrm{abs}} \\
(\mathrm{nm})^{(\mathrm{a})}\end{array}$ & $\begin{array}{l}\lambda_{\mathrm{RTPL}} \\
(\mathrm{nm})^{(\mathrm{b})}\end{array}$ & $\begin{array}{l}\text { FWHM } \\
(\mathrm{nm})^{(\mathrm{b})}\end{array}$ & $\begin{array}{c}\mathrm{S}_{1} \\
(\mathrm{eV})^{(\mathrm{c})}\end{array}$ & $\begin{array}{c}\mathrm{T}_{1} \\
(\mathrm{eV})^{(\mathrm{d})}\end{array}$ & $\begin{array}{c}\Delta \mathrm{EST} \\
(\mathrm{eV})^{(\mathrm{e})}\end{array}$ & $\begin{array}{c}\tau_{\mathrm{p}} \\
(\mathrm{ns})^{(\mathrm{f})}\end{array}$ & $\begin{array}{c}\tau_{\mathrm{d}} \\
(\mu \mathrm{s})^{(\mathrm{g})}\end{array}$ & $\begin{array}{l}\text { HOMO } \\
(e V)^{(i)}\end{array}$ & $\begin{array}{l}\text { LUMO } \\
(\mathrm{eV})^{(\mathrm{j})}\end{array}$ & $\begin{array}{c}\text { Band } \\
\text { gap } \\
(\mathrm{eV})^{(\mathrm{k})}\end{array}$ & $\begin{array}{l}\text { PLQY } \\
(\%)^{(1)}\end{array}$ \\
\hline $\begin{array}{c}p \text { MDBA- } \\
\text { DI }\end{array}$ & 384 & 460 & 59 & 2.99 & 2.92 & 0.07 & 24.6 & $\begin{array}{c}1.60 \\
(0.75)^{(\mathrm{h})}\end{array}$ & 5.50 & 2.61 & 2.89 & 97.8 \\
\hline $\begin{array}{c}m \text { MDBA- } \\
\text { DI }\end{array}$ & 371 & 451 & 52 & 3.04 & 2.92 & 0.12 & 28.0 & $\begin{array}{c}1.90 \\
(0.80)^{(\mathrm{h})}\end{array}$ & 5.48 & 2.57 & 2.91 & 97.3 \\
\hline
\end{tabular}


$t-\mathrm{Bu}-v-$

DABNA

(a) UV-Vis absorption wavelengths measured in $10^{-5} \mathrm{M}$ of toluene solution. (b) PL max emission measured in toluene at $300 \mathrm{~K}$. (c) Calculated by the onset of RTPL spectrum in toluene. (d) Calculated by the onset of low temperature PL (LTPL) spectrum in toluene at $77 \mathrm{~K}$ with $30 \mathrm{~ms}$ of delaying. (e) $\Delta \mathrm{E}_{\mathrm{ST}}=\mathrm{S}_{1}-\mathrm{T}_{1}$. (f) Prompt decay lifetime in toluene. (g) Delayed decay lifetime in toluene. (h) Delayed decay lifetime in methylene chloride. (i) HOMO level energy measured by $\mathrm{CV}$ method. (j) LUMO=HOMO-optical band gap. (k) Calculated by the edge of the absorption spectrum (l) Measured in DBFPO film. $p$ MDBA-DI and $m$ MDBA-DI were $30 \%$ doped and $t$-Bu-v-DABNA was 5\% doped.

(a)

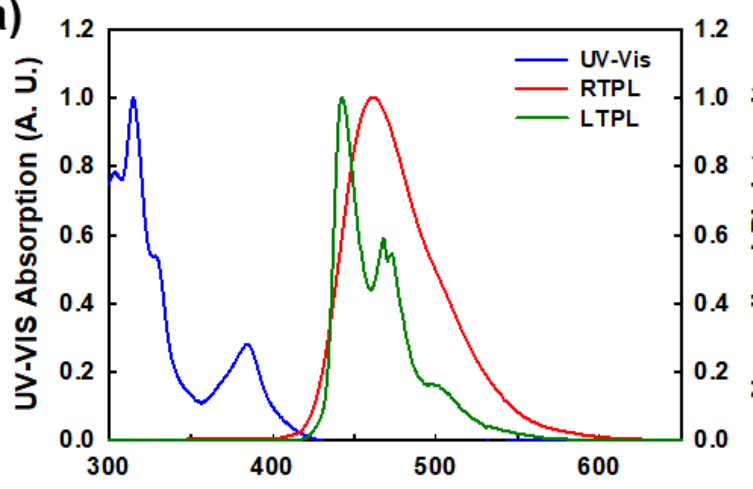

(b)

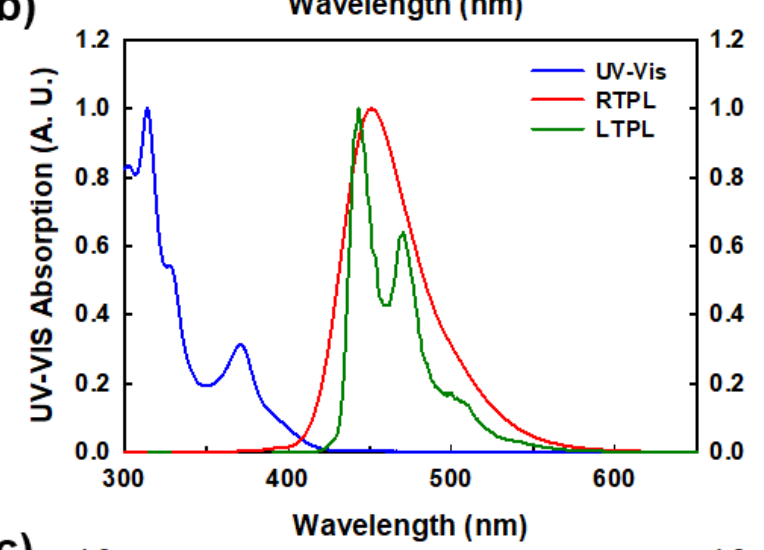

(c)

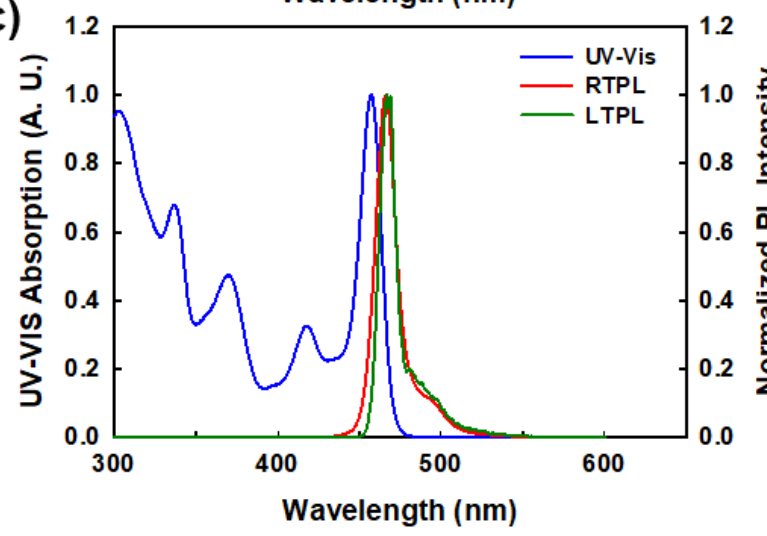

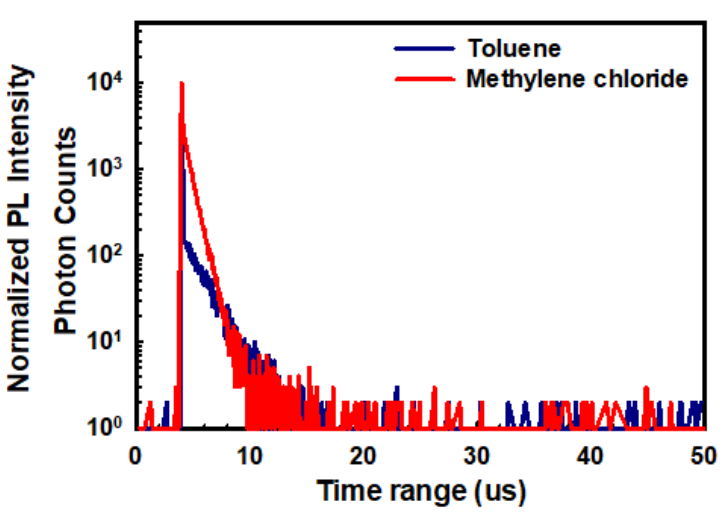
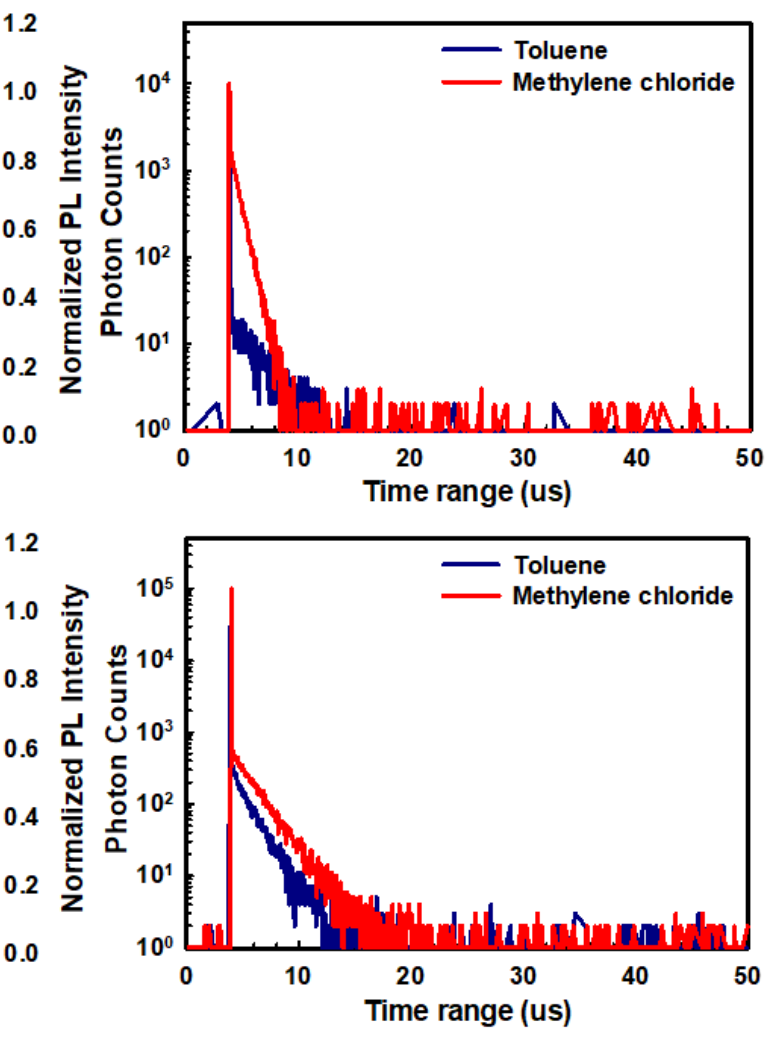

Figure 1. Photophysical properties of synthesized material. Normalized UV-Vis absorption, RTPL, 
and LTPL in 77K toluene, and Transient PL decay both in toluene and methylene chloride of (a) $p$ MDBA-DI, (b) $m$ MDBA-DI, and (c) $t$-Bu- $v$-DABNA.

\section{Energy transfer study}

Efficient FRET process is a key parameter to activate an effective HF system. Generally, FRET rate can be expressed as equation (1). ${ }^{25}$

$k_{F R E T}=\frac{\Phi_{D} \kappa^{2}}{\tau_{D} R^{6}} \frac{9000(\ln 10)}{128 \pi^{5} N_{A} n^{4}} \int_{0}^{\infty} \int F_{D}(\lambda) \epsilon_{A}(\lambda) \lambda^{4} d \lambda$

Where, $\mathrm{R}$ is the intermolecular distance between donor and acceptor. $\Phi_{D}$ stands for the PLQY of donor without acceptor. $F_{D}(\lambda)$ is the normalized emission spectra of the donor and $\epsilon_{A}(\lambda)$ is the molar absorption coefficient of the acceptor. It is obvious that $k_{F R E T}$ mainly depends on the PLQY

of donor and spectral overlap between the absorption of acceptor and PL emission of donor. Since both materials exhibited similar PLQYs, the extent of spectral overlap mainly determines $k_{F R E T}$. Thus, the extent of spectral overlap between $p$ MDBA-DI and $m$ MDBA-DI with $t$-Bu- $v$-DABNA in the DBFPO film state were measured. As a result, absorption spectrum of $t$-Bu- $v$-DABNA was largely overlapped with PL emission spectra of both $p$ MDBA-DI and $m$ MDBA-DI compared to DBA-DI as illustrated in Figure S8, so high $k_{F R E T}$ can be expected from $p$ MDBA-DI and $m$ MDBA-DI. We can notice that more blue shifted $m$ MDBA-DI has larger spectral overlapping with $t$-Bu- $v$-DABNA, and more efficient FRET process would be expected in HF system. To see more clear evidence for good energy transfer, exciton decay curves of $30 \%$ of TADF materials with and without $1 \%$ of $t$-Bu-v-DABNA in DBFPO film were investigated. Additional $1 \%$ of $t$ $\mathrm{Bu}-\mathrm{v}$-DABNA doping, the prompt decay lifetime was shortened with reducing the delayed portion as illustrated in Figure S9. The prompt decay time of $p$ MDBA-DI with $1 \%$ of $t$-Bu- $v$-DABNA 
was reduced from 45.6 to $15.8 \mathrm{~ns}$ and in which $m \mathrm{MDBA}-\mathrm{DI}$ was reduced from 47.0 to $10.1 \mathrm{~ns}$. This means that faster FRET process from TADF assistant host to $t$-Bu- $v$-DABNA accelerated the overall prompt energy transfer process, indicating efficient FRET process. The exact rate constants of FRET in HF system were calculated by using following equation (2). ${ }^{25-26}$

$k_{F R E T}=k_{P F}-k_{r, S}-k_{I S C}$

Where $k_{r, S}$ and $k_{I S C}$ are singlet radiative rate constant, and intersystem crossing rate constant, respectively. The calculated $k_{F R E T}$ of $30 \% m \mathrm{MDBA}-\mathrm{DI}$ and $p \mathrm{MDBA}-\mathrm{DI}$ with $1 \%$ of $t$-Bu- $v$ DABNA was $7.77 \times 10^{7} \mathrm{~s}^{-1}$ and $4.14 \times 10^{7} \mathrm{~s}^{-1}$, respectively. These $k_{F R E T}$ rates are much faster than calculated $k_{r, S}, k_{I S C}$ values (provided Table S1) without $t$-Bu- $v$-DABNA doping, which is an experimental evidence that FRET energy transfer is enhanced in our HF system. The measured TRPL decay lifetime and calculated FRET rate constants are listed in Table S2.

\section{Device Performances}

To understand the TADF characteristics of synthesized materials, the TADF-OLEDs with synthesized materials were fabricated using previously reported device configuration ${ }^{19}$; ITO (50 nm) / HATCN (7 nm) / TAPC (50 nm) / DCDPA (10 nm) / DBFPO: 30\% pMDBA-DI and $m \mathrm{MDBA}-\mathrm{DI}$ or $5 \%$ of $t$-Bu- $v$-DABNA $(25 \mathrm{~nm}) / \mathrm{DBFPO}(5 \mathrm{~nm}) / \mathrm{TPBi}(20 \mathrm{~nm}) / \mathrm{LiF}(1.5 \mathrm{~nm}) /$ Al (100 nm) were fabricated. Indium tin oxide (ITO) and aluminum (Al) were utilized as anode and cathode, respectively. Dipyrazino[2,3-f:2',3'-h]quinoxaline-2,3,6,7,10,11-hexacarbonitrile (HATCN) and lithium fluoride (LiF) were used as hole- and electron injection layers, respectively. 1,1-Bis[4-[N,N'-di(ptolyl)amino]-phenyl] cyclohexane (TAPC) and 1,3,5-tris(1-phenyl-1Hbenzo[d]imidazole-2-yl)benzene (TPBi) served as hole- and electron-transporting layers, respectively. 3,5-Di(9H-carbazol-9-yl)-N,N-diphenylaniline (DCDPA) and DBFPO were utilized 
as exciton blocking layers on the each sides of the EML owing to their higher triplet energy levels. The DBFPO also served as a host material. The maximum electroluminescence (EL) peak at 1,000 $\mathrm{cd} / \mathrm{m}^{2}$ of $p$ MDBA-DI was $483 \mathrm{~nm}$, whereas, $m$ MDBA-DI exhibited of $474 \mathrm{~nm}$, and $m$ MDBA-DI showed $9 \mathrm{~nm}$ blue-shifted emission than $p$ MDBA-DI. Measured CIE coordinates were $(0.15,0.31)$ and $(0.14,0.23)$, respectively. TADF devices of $p$ MDBA-DI and $m$ MDBA-DI exhibited high maximum EQE of 33.1 and $32.8 \%$, and the EQE of both devices were still able to maintained 32.3 and $28.4 \%$ (Lambertian assumption) at $1,000 \mathrm{~cd} / \mathrm{m}^{2}$, respectively. Interestingly, $p$ MDBA-DI device showed alleviated efficiency roll-off compared with those of $m$ MDBA-DI, which can be attributed to smaller $\Delta \mathrm{E}_{S T}$ and two times higher $k_{\mathrm{RISC}}$ of $p$ MDBA-DI. Both TADF materials exhibited surprisingly high EQE values, which cannot be fully explained by only high PLQY and good TADF behavior. The molecular orientation factor $(\Theta)$ of $m$ MDBA-DI was measured with previously reported method. ${ }^{28}$ As expected, high orientation factor of 0.82 value was obtained. (Figure S10 (a)) Additionally, TADF performance of $t$-Bu- $v$-DABNA was also investigated. The maximum electroluminescence (EL) peak at $1,000 \mathrm{~cd} / \mathrm{m}^{2}$ was $474 \mathrm{~nm}$ and $\mathrm{FWHM}$ was narrow as $18 \mathrm{~nm}$, following CIE y coordinate was 0.15 . The measured maximum EQE value of $t-\mathrm{Bu}-v-$ DABNA in TADF device was high as $36.3 \%$ (Lambertian assumption) even though PLQY and TADF performances fall behind $p$ MDBA-DI or $m$ MDBA-DI. To understand such high efficiency, the orientation factor of $t$-Bu-v-DABNA was measured and found to be real high as 0.92. (Figure S10 (b)) Such higher value is interpreted due to the rigid and planar structure of $t$-Bu-v-DABNA. In addition, tert-butyl groups in planar structure of $v$-DABNA results in higher PLQY by preventing the aggregation induced self-quenching compared to $v$-DABNA emitter. Owing to these two reasons, high TADF device efficiency of $t$-Bu-v-DABNA could be observed. Despite high efficiency, severe efficiency roll-off was observed on account of low $k_{R I S C}$ (EQE value 
dropped to $16.5 \%$ at $1,000 \mathrm{~cd} / \mathrm{m}^{2}$ ). Detailed device performances of $v$-DABNA and $t$-Bu- $v$ DABNA are illustrated in Figure S11 and Table S3. From the TADF device performances, $p$ MDBA-DI and $m$ MDBA-DI based devices revealed excellent TADF efficiencies and $t$-Bu- $v$ DABNA emitter manifested a narrow FWHM. Consequently, as our energy transfer study suggests that both $p$ MDBA-DI and $m$ MDBA-DI have high $k_{F R E T}$, so all studies denote that our new materials are ideal candidates to form blue HF. Thus, HF-OLEDs were fabricated with 30\% TADF assistant host and $1 \% t$-Bu-v-DABNA emitter in DBFPO host. All the other layers were used without any change of TADF device configuration. Since the HOMO energy levels of three materials are similar, holes are prone to transfer via TADF assistant host materials, while electrons can be trapped into $t$-Bu- $v$-DABNA. However, as shown in the Figure 2(b), the current density curves of TADF and HF device were similar, indicating of almost no charge trapping emission in HF device. HF devices with $p$ MDBA-DI and $m$ MDBA-DI TADF hosts exhibited $474 \mathrm{~nm}$ of maximum EL spectrum with narrow FWHM of $19 \mathrm{~nm}$. Particularly, mMDBA-DI HF device exhibited clear $t$-Bu- $v$-DABNA EL spectrum with minor emission of $m$ MDBA-DI as shown in Figure 2(d). As our expectation from the energy transfer study, a large spectral overlapping and fast energy transfer rate of $m$ MDBA-DI are crucial to get real final emitter spectrum in HF-OLED. Obtained CIE coordinates of $p$ MDBA-DI based HF device was of $(0.13,0.19)$, while those of $m$ MDBA-DI was $(0.12,0.15)$. Different CIE coordinates was derived of the different emission intensity and wavelength of TADF assistant hosts. Surprisingly, $p$ MDBA-DI and $m$ MDBA-DI based HF devices showed much enhanced maximum EQE of 37.6 and $40.7 \%$ than conventional TADF device. At the same time, we noticed that $p$ MDBA-DI and $m$ MDBA-DI based HF devices still remained high EQE of 33.9 and $35.8 \%$ at $1,000 \mathrm{~cd} / \mathrm{m}^{2}$, respectively. The corresponding plot of current density $(J)$, and luminance $(L)$ versus voltage $(V)$, and EQE versus luminance, 
electroluminescence (EL) spectrum of each TADF and HF devices are depicted in Figure 2. Since both TADF assistant hosts and $t$-Bu-v-DABNA have high horizontal orientation factor, the angular distribution of emission became non-Lambertian shape, resulting in an error in the calculated EQE values from Lambertian assumption measurement method. Thereby, we measured the angular distribution of the devices as described in Figure S12, and recalculated the EQE value by integrating all angular-dependent emission. From this calculation, corrected maximum EQEs for $p$ MDBA-DI and $m$ MDBA-DI HF-OLEDs were $36.2 \%$ and $39.1 \%$, respectively.

(a)

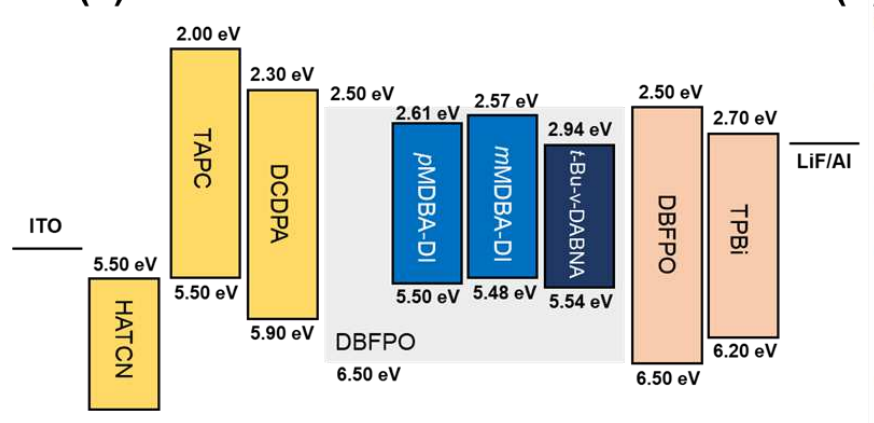

(c)

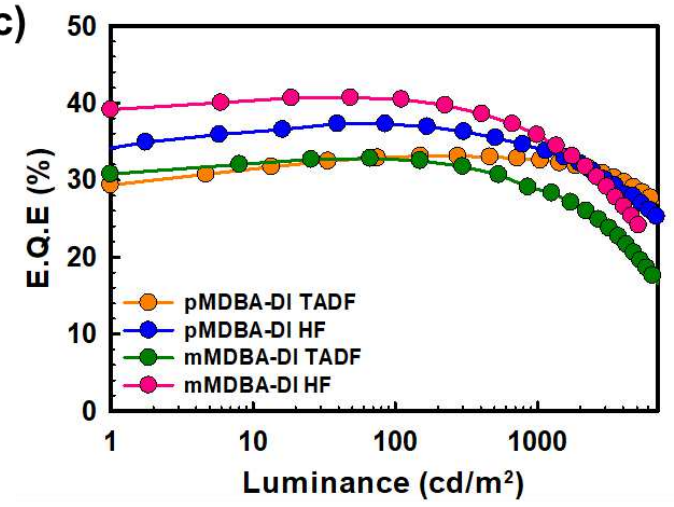

(b)

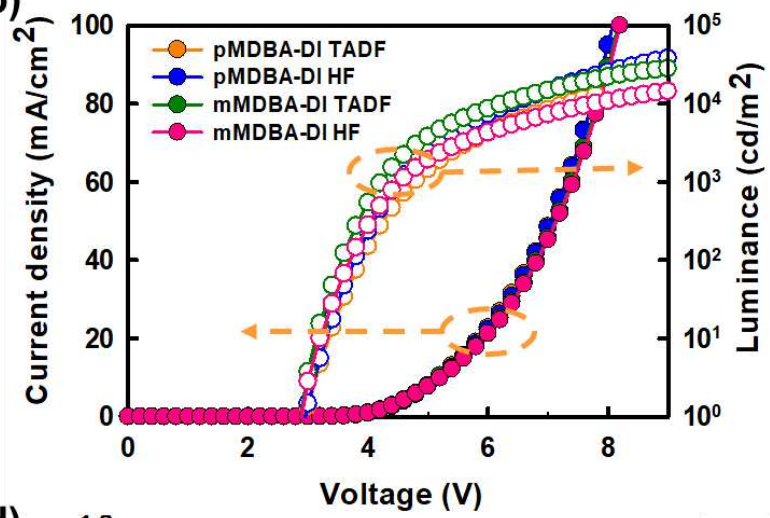

(d)

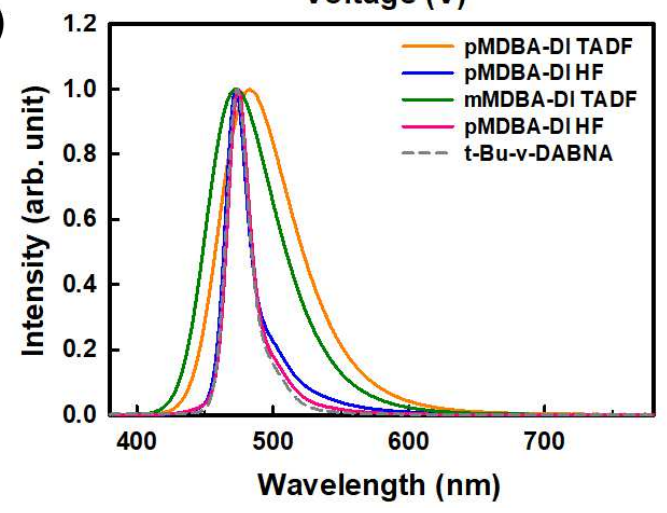

Figure 2. (a) Device configuration and energy-level diagram, (b) Current efficiency verse voltage $(J-V)$ and luminance versus voltage $(L-V)$ curves, (c) EQE value versus luminance of $p$ MDBA-DI and $m$ MDBA-DI of TADF and HF device. (d) EL spectrum of TADF, HF device and $t$-Bu-vDABNA.

\section{Analysis of high device efficiency}


The $p$ MDBA-DI and $m$ MDBA-DI based HF devices obtained much higher maximum EQEs than individual TADF devices. Especially, $m$ MDBA-DI achieved 39.1\% of maximum EQE value with pure blue emission color of $t$-Bu-v-DABNA. To understand the root of such efficiency enhancement in HF device, we analyzed related factors of EQE. Generally, obtained EQE can be expressed by the following equation (3). ${ }^{29}$

$\eta_{\mathrm{EQE}}=\gamma \times \eta_{\mathrm{S} / \mathrm{T}} \times q_{\mathrm{eff}}\left(q_{\mathrm{PL}}, \Theta, \Gamma\right) \times \eta_{\mathrm{out}}(\Theta, \Gamma)$

Where $\gamma$ is the charge balance factor, $\eta_{\mathrm{S} / \mathrm{T}}$ is the singlet-triplet factor, $q_{\mathrm{eff}}$ is the effective quantum yield, and $\eta_{\text {out }}$ is the out-coupling efficiency of the emitted light. The $\eta_{\text {out }}$ is not only influenced by the device structure, but also by the horizontal orientation factor of the emitter. High out-coupling efficiency can effectively help improving device efficiency compared to isotropic emitters ( $\eta_{\text {out }}$ $0.2 \sim 0.3)$. Considering the high orientation value of 0.82 of $m$ MDBA-DI, the $\eta_{\text {out }}$ value was calculated to be 0.350 in this device by the optical simulation (used Setfos 4.3 program). Since the orientation value of $t$-Bu-v-DABNA was measured to be $0.92, \eta_{\text {out }}$ value could be higher value. Calculated possible device efficiency was $37.8 \%$ as shown in Figure 3. Thus, $6 \%$ of EQE value was enhanced by the assistance of increased orientation factor of $t$-Bu- $v$-DABNA. However, such molecular orientation effect cannot be fully explained by the $39.1 \%$ of high efficiency. So, low doing concentration merit of HF device was considered. When the device doping percentage decreased from 5\% to 1\%, PLQY tends to increase. Our measured PLQY was increased from 91.9\% to $93.1 \%$ due to reduction of self-quenching. This PLQY increase could enhance our device EQE additionally about $0.5 \%$ in our HF system. Although all possible way of EQE enhancement were considered, our device EQE is still high about $0.8 \%$. Therefore, additional singlet exciton generation on $t$-Bu- $v$-DABNA was taken into consideration, because $t$-Bu- $v$-DABNA has TADF characteristics and can harvest more singlet excitons via RISC of triplet excitons formed by direct 
charge trapping or DET from the host. When TADF is used as assistant host, the total internal EL efficiency $\left(\phi_{E L(i n t)}\right)$ can be expressed by following equation (4). ${ }^{30-31}$

$\phi_{E L(i n t)}=\left[\eta_{0}\left(S_{1}\right)+\frac{\left(\eta_{0}\left(S_{1}\right) \phi_{I S C, H}+\eta_{0}\left(T_{1}\right)\right) \phi_{R I S C}}{1-\phi_{I S C, H} \phi_{R I S C}}\right] \phi_{F R E T} \phi_{P L, D}$

Where, $\eta_{0}\left(S_{1}\right)$ is the singlet exciton ratio, and $\eta_{0}\left(T_{1}\right)$ is the triplet exciton ratio. $\phi_{I S C, H}$ is the ISC efficiency of the TADF assistant host material, and $\phi_{P L, D}$ stands for the PL quantum efficiency of the emitter. $\phi_{F R E T}$ is the FRET efficiency from TADF assistant host to fluorescence emitter. Due to the large spectral overlap, high $\phi_{F R E T}$ was possible, $t$-Bu- $v$-DABNA was able to receive large amount of $m$ MDBA-DI recycled singlet excitons. Simultaneously, $t$-Bu-v-DABNA can harvest additional singlet exciton through triplet exciton recycling via RISC process from trapping or DET. Considering both energy transfer and triplet exciton recycling by $t$-Bu- $v$-DABNA, internal quantum efficiency $\left(\phi_{E L(i n t)}\right)$ of $t$-Bu-v-DABNA HF system can be described by the following equation (5).

$\phi_{E L(i n t)}=\left[\left(\eta_{0}\left(S_{1}\right)+\frac{\left(\eta_{0}\left(S_{1}\right) \phi_{I S C, H}+\eta_{0}\left(T_{1}\right)\right) \phi_{R I S C}}{1-\phi_{I S C, H} \phi_{R I S C}}\right) \phi_{F R E T}+\frac{\eta_{0}\left(T_{1}\right)_{D E T} \phi_{R I S C}}{1-\phi_{I S C} \phi_{R I S C}}+\frac{\eta_{0}\left(T_{1}\right)_{T R A P} \phi_{R I S C}}{1-\phi_{I S C} \phi_{R I S C}}\right] \phi_{P L, D}$

Where, $\eta_{0}\left(T_{1}\right)_{D E T}$ and $\eta_{0}\left(T_{1}\right)_{T R A P}$ represents the triplet exciton occurred from DET and trapping, respectively. $\frac{\eta_{0}\left(T_{1}\right)_{D E T} \phi_{R I S C}}{1-\phi_{I S C} \phi_{R I S C}}$ and $\frac{\eta_{0}\left(T_{1}\right)_{T R A P} \phi_{R I S C}}{1-\phi_{I S C} \phi_{R I S C}}$ are recycled additional singlet exciton fraction from $t$-Bu- $v$-DABNA via DET or trapping emission. In our system, the remaining $0.8 \%$ of EQE enhancement may originated by this effect. The trace of HF device efficiency increment is illustrated in Figure 3. On the other hand, $p$ MDBA-DI TADF exhibited the less effective device efficiency improvement in HF system. Due to less spectrum overlap, $k_{F R E T}$ was relatively slower 
and singlet emission from TADF assistant host was enlarged in total HF EL spectrum, thus, the improvement of $\eta_{\text {out }}$ value was limited.

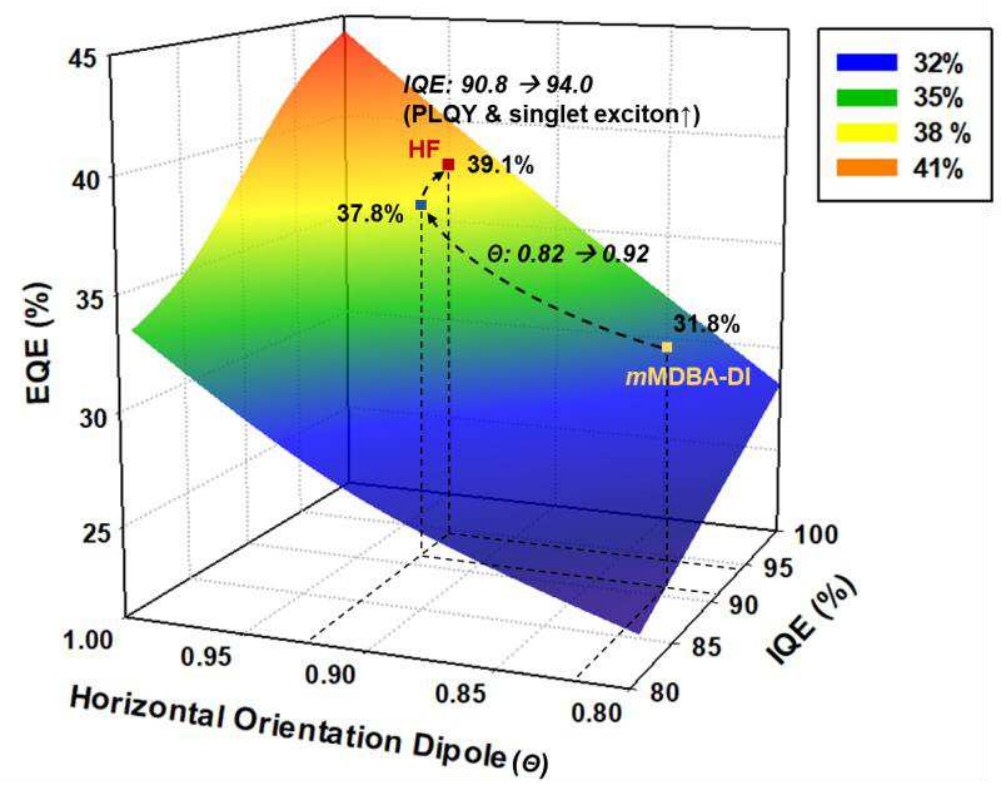

Figure 3. Trace of device efficiency increment from $m$ MDBA-DI TADF device to HF device. $\left(\mathrm{IQE}=\boldsymbol{\eta}_{\mathbf{S} / \mathbf{T}} \times \boldsymbol{q}_{\mathbf{P L}}\right)$

Table 2. Device performances of $p$ MDBA-DI and $m$ MDBA-DI TADF devices and HF devices with $t$-Bu- $v$-DABNA.

\begin{tabular}{|c|c|c|c|c|c|c|c|}
\hline & $\begin{array}{l}\text { Turn on } \\
\text { voltage }{ }^{(a)} \\
\text { (V) }\end{array}$ & $\begin{array}{l}\text { Driving } \\
\text { voltage }^{(b)} \\
\text { (V) }\end{array}$ & $\begin{array}{c}\text { Current } \\
\text { Efficiency }(\mathrm{Cd} / \mathrm{A}) \\
(\operatorname{Max} / 1,000 \\
\left.\mathrm{cd} / \mathrm{m}^{2}\right)\end{array}$ & $\begin{array}{c}\text { EQE }(\%) \\
(\operatorname{Max} / 1,000 \\
\left.\mathrm{cd} / \mathrm{m}^{2}\right)\end{array}$ & $\begin{array}{l}\text { Max } \\
\text { emission } \\
\text { peak } \\
(\mathrm{nm})\end{array}$ & $\begin{array}{l}\text { FWHM } \\
(\mathrm{nm})\end{array}$ & $\begin{array}{c}\text { CIE } \\
\text { coordinates } \\
(1,000 \\
\left.\mathrm{cd} / \mathrm{m}^{2}\right)\end{array}$ \\
\hline $\begin{array}{c}p \text { MDBA-DI } \\
\text { TADF }\end{array}$ & 2.9 & 4.8 & $\begin{array}{c}57.3 / 55.3 \\
(54.9 / 53.4)^{(\mathrm{c})}\end{array}$ & $\begin{array}{c}33.1 / 32.3 \\
(32.0 / 30.8)^{(\mathrm{c})}\end{array}$ & 483 & 60 & $(0.15,0.31)$ \\
\hline $\begin{array}{c}p \text { MDBA-DI } \\
\text { HF }\end{array}$ & 2.9 & 4.8 & $\begin{array}{c}39.4 / 36.0 \\
(37.9 / 34.8)^{(\mathrm{c})}\end{array}$ & $\begin{array}{c}37.6 / 33.9 \\
(36.2 / 33.0)^{(\mathrm{c})}\end{array}$ & 474 & 19 & $(0.13,0.19)$ \\
\hline $\begin{array}{l}m \mathrm{MDBA}- \\
\text { DI TADF }\end{array}$ & 2.9 & 4.5 & $\begin{array}{c}39.4 / 33.8 \\
(38.2 / 32.8)^{(\mathrm{c})}\end{array}$ & $\begin{array}{c}32.8 / 28.4 \\
(31.8 / 27.9)^{(\mathrm{c})}\end{array}$ & 474 & 60 & $(0.14,0.23)$ \\
\hline
\end{tabular}


mMDBA-

DI HF
$2.9 \quad 4.5$

4.5

$31.6 / 27.7$

$(30.3 / 26.6)^{(c)}$
$40.7 / 35.8$

$(39.1 / 34.3)^{(\mathrm{c})}$
474

19

$(0.12,0.15)$

(a)Turn on voltage at $1 \mathrm{~cd} / \mathrm{m}^{2}$, (b) Driving voltage at $1,000 \mathrm{~cd} / \mathrm{m}^{2}$, (c) Calculated value by integrating all angulardependent emission.

\section{Conclusion}

In conclusion, two new highly efficient blue organo-boron TADF materials and narrow spectral width $t$-Bu- $v$-DABNA emitter was designed and synthesized for efficient and pure blue HFOLEDs. Both $p$ MDBA-DI and $m$ MDBA-DI exhibited excellent TADF performances with hypsochromic shifted emission from DBA-DI, leading to acquire large spectrum overlap with $t$ Bu- $v$-DABNA emitter. The $t$-Bu- $v$-DABNA exhibited high PLQY and narrow FWHM as a good candidate emitter for an efficient HF-OLEDs. $p$ MDBA-DI and $m$ MDBA-DI based TADF devices exhibited high $\mathrm{EQE}_{\max }$ of 33.1 and $32.8 \%$, respectively. Especially, HF device comprised of $m$ MDBA-DI and $t$-Bu- $v$-DABNA showed significantly high $\mathrm{EQE}_{\max }$ of $40.7 \%$ (Lambertian assumption) along with narrow emission spectra by reflecting the emission of $t$-Bu- $v$-DABNA $(\mathrm{CIE} \mathrm{y}=0.15)$. Additionally, we have quantitatively analyzed the individual contributions to high EQE using the theoretical simulation and photophysical properties. Such high efficiency was dominantly attributed to the following criterions; high horizontal orientation factor, reduced selfquenching while enhancing PLQY and additional singlet exciton recycling process from DET or trapping emission on $t$-Bu- $v$-DABNA emitter. We believe that our design strategy and device analysis would propose a way to attain highly efficient pure blue HF-OLEDs.

Acknowledgement: This work was supported by the National Research Foundation of Korea (Grant No. NRF-2019M3D1A2104019). 
Competing interests: The authors declare no competing interests towards this publication.

Additional information: Supplementary information is available for this paper: Detailed synthetic procedures; NMR spectra; solvatochromic spectra; time resolved PL spectra; TGA and DSC curves; CV curves; optimized geometry of syntheszed materials; device characterization data. 


\section{References:}

1. Uoyama, H., Goushi, K., Shizu, K., Nomura, H., \& Adachi, C. Highly efficient organic lightemitting diodes from delayed fluorescence. Nature 492, 234-238 (2012).

2. Adachi, C. et al. Thermally Activated Delayed Fluorescence from Sn4+-Porphyrin Complexes and Their Application to Organic Light Emitting Diodes- A Novel Mechanism for Electroluminescence. Adv. Mater. 21, $4802-4806$ (2009).

3. Hirata, S. et al. Highly efficient blue electroluminescence based on thermally activated delayed fluorescence. Nature Materials 14, 330-336 (2015).

4. Yokoyama, M., Inada, K., Tsuchiya, Y., Nakanotani, H., \& Adachi, C. Trifluoromethane modification of thermally activated delayed fluorescence molecules for high-efficiency blue organic light-emitting diodes. Chem. Commun. 54, 8261-8264 (2018).

5. Lee, J.-H. et al. Blue organic light-emitting diodes: current status, challenges, and future outlook. J. Mater. Chem. C. 7, 5874-5888 (2019).

6. Zhang, Q. et al. Design of Efficient Thermally Activated Delayed Fluorescence Materials for Pure Blue Organic Light Emitting Diodes. J. Am. Chem. Soc. 134, 14706-14709 (2012).

7. Lim, H. et al. Highly Efficient Deep-Blue OLEDs using a TADF Emitter with a Narrow Emission Spectrum and High Horizontal Emitting Dipole Ratio. Adv. Mater. 32, 2004083 (2020).

8. Kim, J. U. et al. Nanosecond-time-scale delayed fluorescence molecule for deep-blue OLEDs with small efficiency roll off. Nat. Commun. 11, 1765 (2020). 
9. Nakanotani, H. et al. High-efficiency organic light-emitting diodes with fluorescent emitters. Nat. Commun. 5, 4016 (2014)

10. Kim, K.-H., Moon, C.-K., Sun, J.W., Sim, B., \& Kim, J.-J. Triplet Harvesting by a Conventional Fluorescent Emitter Using Reverse Intersystem Crossing of Host Triplet Exciplex. Adv. Opt. Mater. 3, 895-899 (2015).

11. Byeon, S. Y., Lee, D. R., Yook, K. S., \& Lee, J. Y. Recent Progress of Singlet-ExcitonHarvesting Fluorescent Organic Light-Emitting Diodes by Energy Transfer Processes. Adv. Mater. 31, 1803714 (2019).

12. Ahn, D. H., Jeong, J. H., Song, J., Lee, J. Y., \& Kwon, J. H. Highly Efficient Deep Blue Fluorescent Organic Light-Emitting Diodes Boosted by Thermally Activated Delayed Fluorescence Sensitization. ACS Appl. Mater. Interfaces. 10, 10246-10253 (2018).

13. Adachi, J., Kakizoe, H., Tsang, P. K. D. \& Endo, A. 10.1: Invited Paper: Hyperfluorescence ${ }^{\text {TM}}$; a Game Changing Technology of OLED Display. SID Symposium Digest of Technical Papers 50, 95-98 (2019).

14. Zhang, D., Song, X., Cai, M. \& Duan, L. Blocking Energy-Loss Pathways for Ideal Fluorescent Organic Light-Emitting Diodes with Thermally Activated Delayed Fluorescent Sensitizers. Adv. Mater. 30, 1705250 (2018).

15. Song, W., Lee, I. \& Lee, J. Y. Host Engineering for High Quantum Efficiency Blue and White Fluorescent Organic Light-Emitting Diodes. Adv. Mater. 27, 4358-4363 (2015).

16. Kondo, Y. et al. Narrowband deep-blue organic light-emitting diode featuring an organoboron-based emitter. Nat. Photon. 13, 678-682 (2019). 
17. Chan, C.-Y. et al. Stable pure-blue hyperfluorescence organic light-emitting diodes with highefficiency and narrow emission. Nat. Photon. 15, 203-207 (2021).

18. Jeon, S.O. et al. High-efficiency, long-lifetime deep-blue organic light-emitting diodes. Nat. Photon. 15, 208-215 (2021).

19. Ahn, D. H. et al. Highly efficient blue thermally activated delayed fluorescence emitters based on symmetrical and rigid oxygen-bridged boron acceptors. Nat. Photon. 13, 540-546 (2019).

20. Ahn, D. H. et al. Rigid Oxygen-Bridged Boron-Based Blue Thermally Activated Delayed Fluorescence Emitter for Organic Light-Emitting Diode: Approach towards Satisfying High Efficiency and Long Lifetime Together. Adv. Opt. Mater. 8, 2000102 (2020).

21. Hirai, H. et al. One-Step Borylation of 1,3-Diaryloxybenzenes Towards Efficient Materials for Organic Light-Emitting Diodes. Angew. Chem. Int. Ed. 54, 13581-13585 (2015).

22. Karthik, D. et al. Highly efficient blue thermally activated delayed fluorescence organic light emitting diodes based on tercarbazole donor and boron acceptor dyads. J. Mater. Chem. C. 8, 2272-2279 (2020)

23. Schrödinger, LLC, New York, (2019)

24. Zhang, Q. et al. Efficient blue organic light-emitting diodes employing thermally activated delayed fluorescence. Nat. Photon. 8, 326-332 (2014).

25. Song, X., Zhang, D., Zhang, Y., Lu, Y. \& Duan, L. Strategically Modulating Carriers and Excitons for Efficient and Stable Ultrapure-Green Fluorescent OLEDs with a Sterically Hindered BODIPY Dopant. Adv. Opt. Mater. 8, 2000483 (2020). 
26. Zhang, D. et al. Efficient and Stable Deep-Blue Fluorescent Organic Light-Emitting Diodes Employing a Sensitizer with Fast Triplet Upconversion. Adv. Mater. 32, 1908355 (2020).

27. Jung, Y. H. et al. A New BODIPY Material for Pure Color and Long Lifetime Red Hyperfluorescence Organic Light-Emitting Diode. ACS Appl. Mater. Interfaces. 13, 17882$17891(2021)$.

28. Ko, I. J. et al. An accurate measurement of the dipole orientation in various organic semiconductor films using photoluminescence exciton decay analysis. Phys. Chem. Chem. Phys. 21, 7083-7089 (2019).

29. Kim, S.-Y. et al. Organic Light-Emitting Diodes with 30\% External Quantum Efficiency Based on a Horizontally Oriented Emitter. Adv. Funct. Mater. 23, 3896-3900 (2013).

30. Kim, H.-G., Kim, K.-H., Moon, C.-K., \& Kim, J.-J. Harnessing Triplet Excited States by Fluorescent Dopant Utilizing Codoped Phosphorescent Dopant in Exciplex Host for Efficient Fluorescent Organic Light Emitting Diodes. Adv. Opt. Mater. 5, 1600749 (2017).

31. Zhang, D. et al. High-Efficiency Fluorescent Organic Light-Emitting Devices Using Sensitizing Hosts with a Small Singlet-Triplet Exchange Energy. Adv. Mater. 26, 5050-5055 (2014). 


\section{Supplementary Files}

This is a list of supplementary files associated with this preprint. Click to download.

- RevisedSIv4.docx 